\title{
Strain Characterization of Suspended-Core Fiber Tapers
}

\author{
Ricardo M. André ${ }^{\mathrm{a}, \mathrm{b}}$, Susana O. Silva ${ }^{\mathrm{a}, \mathrm{b}}$, Martin Becker ${ }^{\mathrm{c}}$, Kay Schuster ${ }^{\mathrm{c}}$, M. Rothardt ${ }^{\mathrm{c}}$, H. Bartelt ${ }^{\mathrm{c}}$, \\ Manuel B. Marques ${ }^{\mathrm{a}, \mathrm{b}}$, Orlando Frazão*a \\ ${ }^{a}$ INESC Porto, Rua do Campo Alegre 687, 4169-007 Porto, Portugal; \\ ${ }^{\mathrm{b}}$ Departamento de Física da Faculdade de Ciências da Universidade do Porto, Rua do Campo Alegre \\ 687, 4169-007 Porto, Portugal \\ ${ }^{c}$ IPHT, Institute of Photonic Technology, Jena, Albert-Einstein-Str. 9, 07745 Jena, Germany
}

\begin{abstract}
Suspended core fiber tapers with different cross sections (from $70 \mu \mathrm{m}$ to $120 \mu \mathrm{m}$ diameter) were produced by filament heating. Before obtaining the taper, the spectral behavior of the suspended core fiber presents multimode interference. When the taper is made an intermodal interference is observed. This effect is clearly visible for high taper reduction. The spectral response of the microtaper inside the suspended core fiber is similar to a beat of two interferometers. The microtaper was subjected to strain, and an increase of sensitivity with the reduction of the transverse area was observed. When the taper was immersed in liquids with different refractive indices or subjected to temperature variations, no spectral change occurred.
\end{abstract}

Keywords: Optical fiber sensor, taper, microstructured fiber

\section{INTRODUCTION}

Optical fibers based on silica have become an irreplaceable asset in telecommunications, data transport and sensing. Low loss single mode fibers (SMFs) have revolutionized telecommunications but fall short when much smaller dimensions are needed for integrated photonic devices. Tapering of conventional fibers to reduce the waveguide dimensions has been thoroughly investigated. Taper adiabaticity and shape have been extensively studied [1,2]. Many tapering techniques have been developed such as pulling the fiber around a heated sapphire rod [3], $\mathrm{CO}_{2}$ lasers [4], and flamebrushing [5]. Low-loss tapers with sub-wavelength diameters offer empowering optical and mechanical properties such as strong confinement and consequent non-linear interactions [6], large evanescent fields [5], and high mechanical strength [7].

In the last 15 years, microstructured optical fibers have attracted a lot of attention due to their excellent properties. Over the last decade, tapering of microstructured optical fibers has been enhanced $[8,9]$ and very interesting optical microstructures have been developed. It was shown that microstructured optical fibers can be tapered while retaining their cross-sectional profile with little or no distortion [10]. The cross-sectional profile can thus be scaled down proportionally producing structures with very small core diameters. Magi et al. [10] were the first to achieve a pitch below $300 \mathrm{~nm}$ in a tapered holey fiber. Tapered holey fiber as a temperature-independent strain sensor was also reported [11]. Tapered microstructured optical fibers have also been employed for efficient supercontinuum generation [6].

In this paper, the tapering of a suspended core fiber (SCF) is investigated. In particular, a SCF with a small core diameter was tapered down reducing even more its diameter. Tapers of different cross-section diameters were produced by filament heating and were characterized in strain, temperature, and external refractive index.

*rmeloandre@alunos.fc.up.pt

Microstructured and Specialty Optical Fibres, edited by Kyriacos Kalli, Alexis Mendez,

Proc. of SPIE Vol. 8426, 84260G · (C) 2012 SPIE · CCC code: 0277-786X/12/\$18 · doi: 10.1117/12.922812

Proc. of SPIE Vol. 8426 84260G-1 


\section{TAPER PRODUCTION}

Figure 1 illustrates the experimental setup used to produce and characterize the SCF tapers. The tapers were produced using a VYTRAN-glass processing workstation. The VYTRAN (GPX-3000 series) is a glass processing platform that performs fusion splicing and tapering of specialty fibers. The system consists of a filament heater, precision stages with multi-axis control (fiber holding blocks) and a microscopic high resolution CCD imaging system. The filament heater has a wide temperature range $\left(100{ }^{\circ} \mathrm{C}\right.$ to $\left.3000{ }^{\circ} \mathrm{C}\right)$ which makes it possible to fuse and process various fiber types and sizes. The working principle is based on heating a portion of fiber in the filament heater to its softening point while applying a tensile force by pulling the fiber with the holding blocks. As the fiber elongates, its cross sectional area will be reduced accordingly. Taper properties can be ascertained by means of a graphic interface. The tapers were fabricated in microstructured fiber (IPHT) with $7.2 \mu \mathrm{m}$ and $123 \mu \mathrm{m}$ core and cladding diameters, respectively. This fiber has four holes with diameters of $57 \mu \mathrm{m}$ each and a bridge width of $0.9 \mu \mathrm{m}$ (see Figure 2 (a)). $10 \mathrm{~mm}$-long tapers were made in SCF sections that in turn were spliced between two single-mode fibers (SMF28). A broadband source (BBS) in the 1550 $\mathrm{nm}$ spectral range and an optical spectrum analyzer (OSA) were used to monitor in real time the tapering process. A similar experimental setup was used to characterize the SCF tapers with increasing strain. But in this case a setup without the filament heater was used.

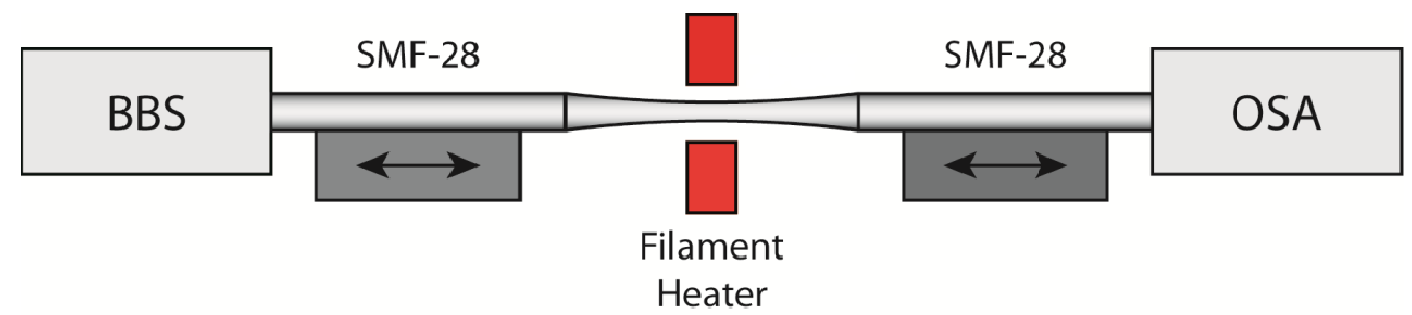

Figure 1. Experimental setup.

One observes from Figure 2 (b) that while the original SCF is tapered, both core and cladding diameters are reduced. It should be noted that the core and cladding have proportional reductions and the cross sectional profile is maintained. Even at $32 \%$ of the original size, the holes do not collapse and little distortion is visible. The ratio between core and cladding diameters is $0.069 \pm 0.002$. Tapers were also immersed in a liquid with a refractive index of 1.36 and no change of spectral response occurred, confirming that the holes had not collapsed. Figure 2 (b) also shows a photo of the taper region which confirms this behavior.

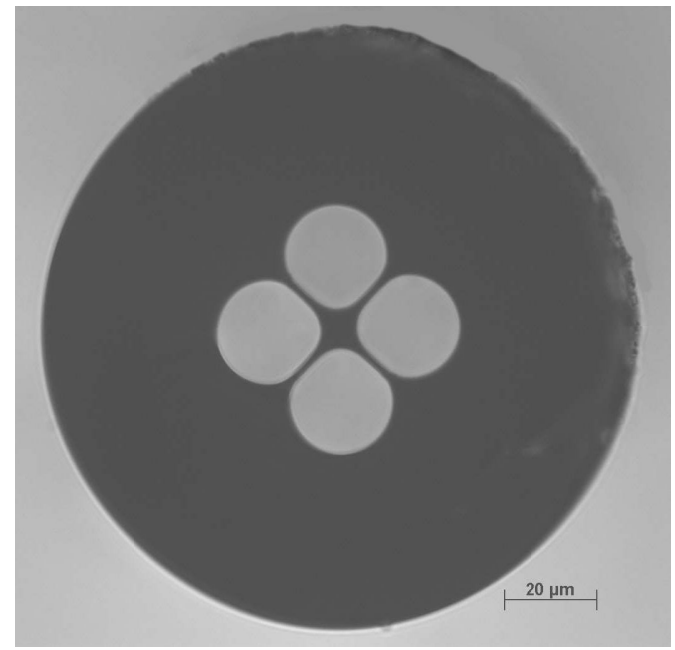

(a)

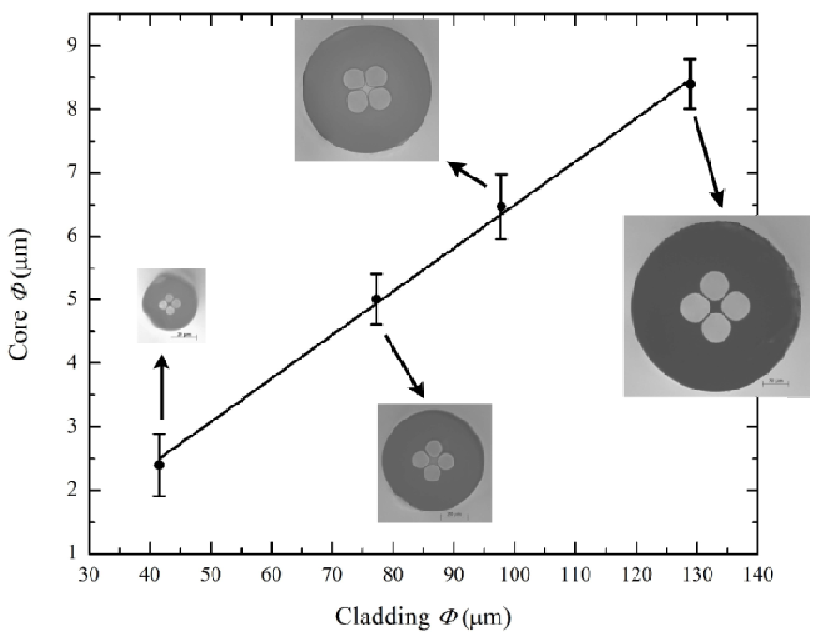

(b)

Figure 2. (a) Suspended core fiber used in this work; (b) Relationship between core and cladding diameters at taper waist. 


\section{SIMULATIONS}

The suspended core fiber shown in Figure 2 was simulated using a Beam Propagation Method (BPM) to obtain the modes supported by such structure. In Figure 3, the first four propagating modes are represented. These modes are evidently orthogonal, but when they recombine at the output splice between the SCF and SMF they interfere with each other [12].

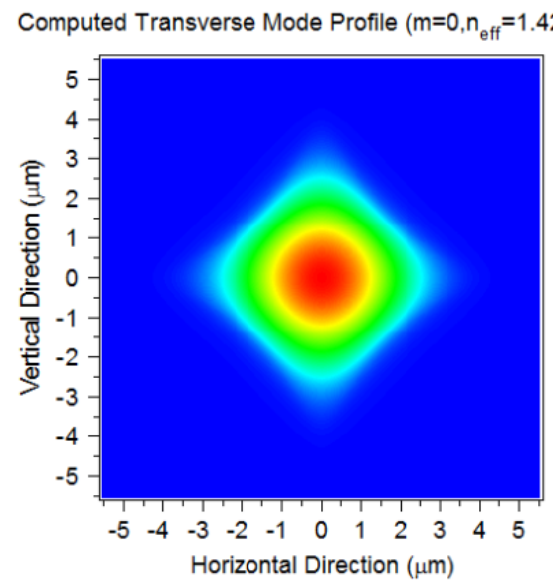

(a)

Computed Transverse Mode Profile $\left(m=2, n_{\text {eff }}=1.40145\right)$

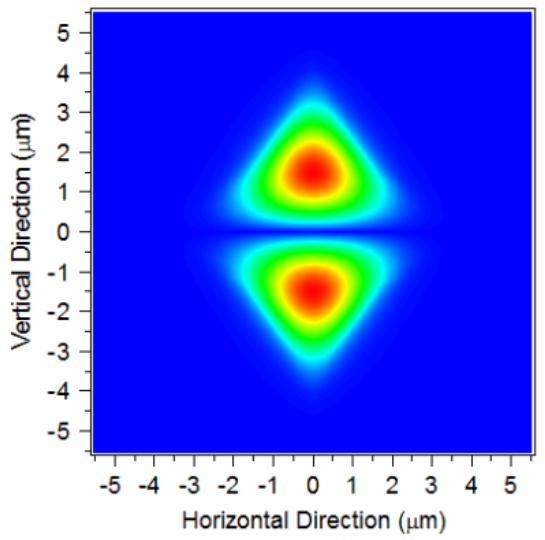

(c)
Computed Transverse Mode Profile $\left(m=1, n_{\text {eff }}=1.40145\right)$
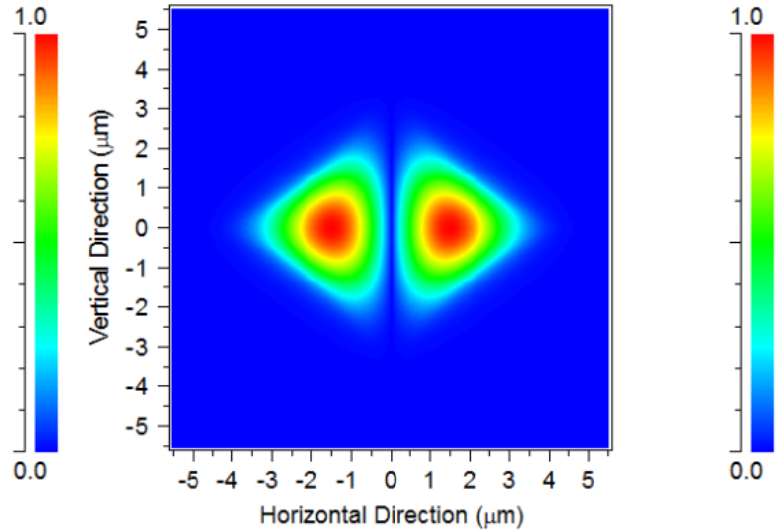

(b)

Computed Transverse Mode Profile $\left(m=3, n_{\text {eff }}=1.381925\right)$
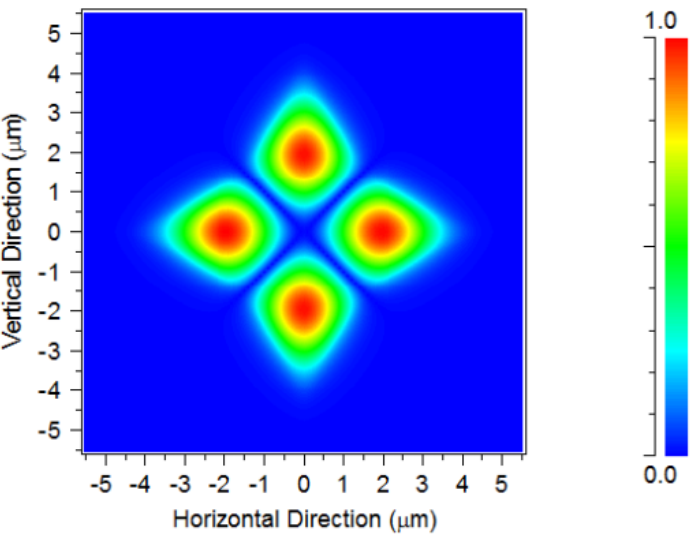

(d)

Figure 3. BPM simulations of the four lowest order modes propagating in the four-hole suspended core fiber. 
When applying the WKB method to multimode graded-index fibers and taking the limit for step-index fibers, one obtains an expression for the number of modes: $N=V^{2} / 2$, valid for cylindrical (core and cladding) fibers with normalized frequencies $(V)$ greater than 5 [13]. Figure 4 shows that the actual number of modes of the SCF structure does follow a quadratic tendency but it does not correspond to the expression previously stated. In fact, the number of modes follows an expression, derived from the quadratic fit of Figure 4, given by $N \approx V^{2} / 5$.

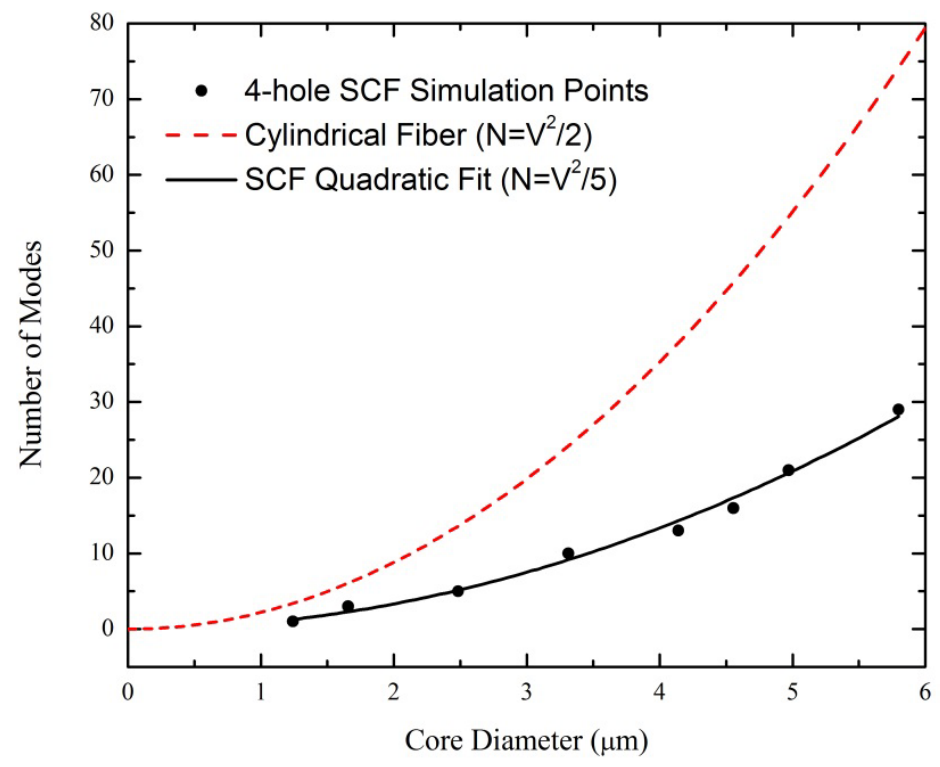

Figure 4. Relationship between the number of modes and the core diameter of a suspended core four-hole fiber. Also included are a quadratic fit (solid) and the theoretical expression for cylindrical fibers (dashed). 


\section{EXPERIMENTAL RESULTS}

Before tapering, the sensing head acts as a multimode interference device - the light field propagating along the input SMF enters the SCF section and several modes are excited. This interference appears because different modes, which propagate along the SCF with different phase velocity, are recombined at the splice between single mode fiber and SCF and, in this case, a well-defined notch peak at $\sim 1585 \mathrm{~nm}$ appears (see Figure 5 (a)). After tapering, intermodal interference appears, as presented in Figure 5 (b). This occurs due to the reduction in core diameter and consequent reduction of the number of propagating modes. Using the expression obtained from the simulations, it is possible to estimate a $70 \%$ reduction in the number of modes when tapering the original SCF to an outer diameter of $70 \mu \mathrm{m}$. Figure 5 (b) shows a new spectral response when compared with Figure 5 (a). A beat signal appears due to the combination of a few interferometers.
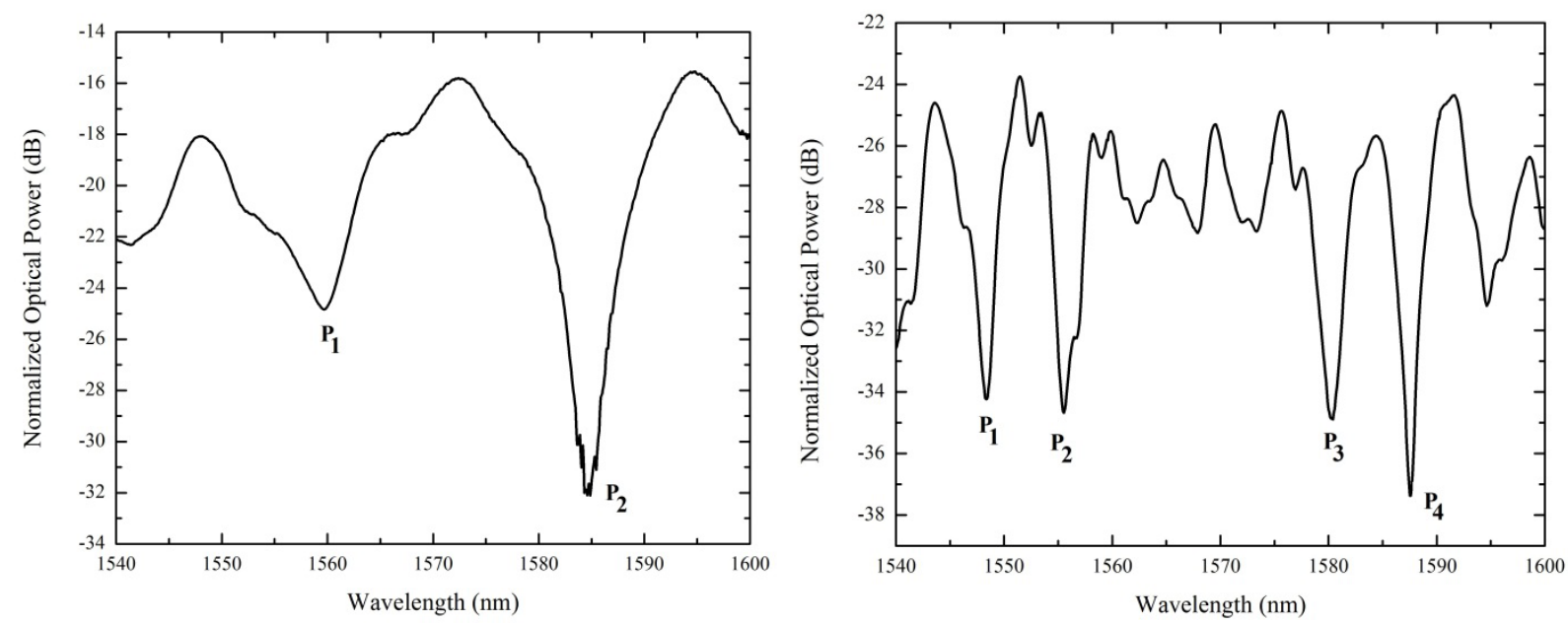

Figure 5. Spectral response a) before and b) after fabricating the $70 \mu \mathrm{m}$ taper in the suspended core fiber.

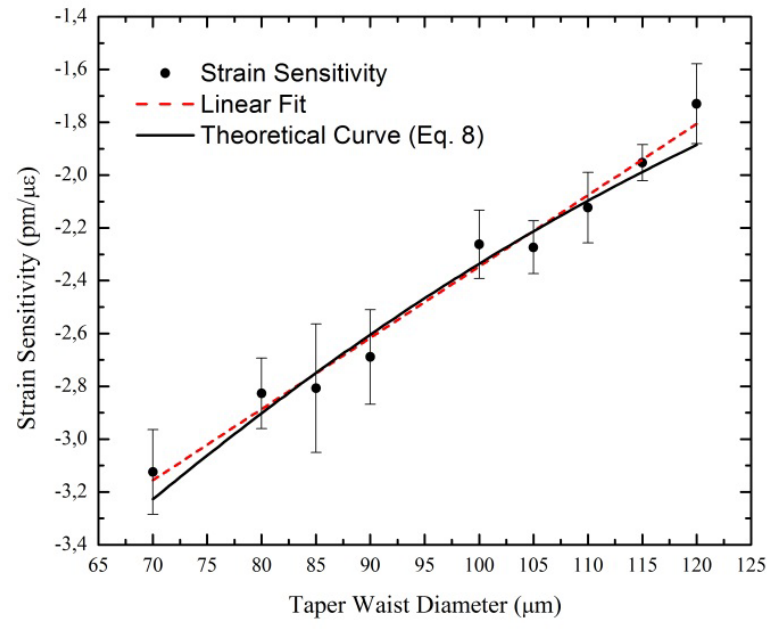

(a)

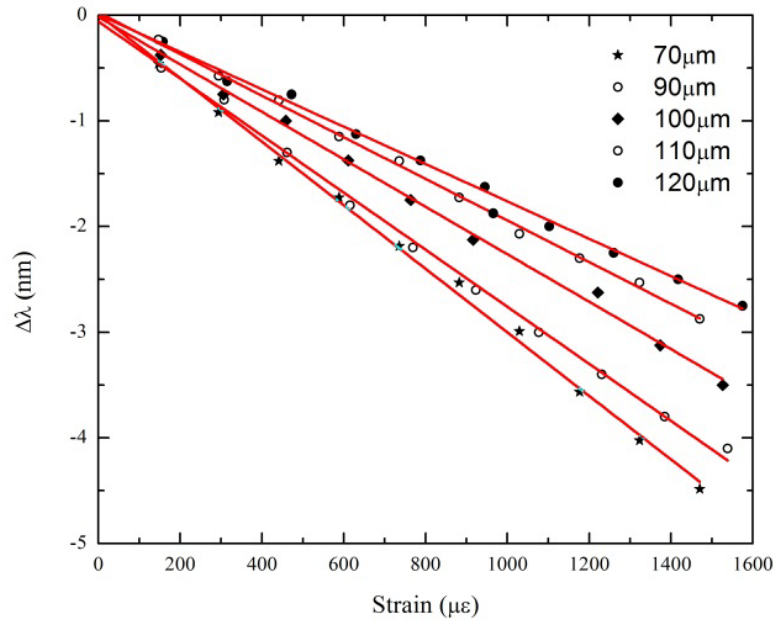

(b)

Figure 6. (a) Relationship between the strain sensitivity and the taper waist; (b) Wavelength change with applied strain for several tapers. 
Several sensing structures were tapered down with different SCF waist diameters, namely, 120, 115, 110, 105, 100, 90, 85,80 and $70 \mu \mathrm{m}$. For strain characterization, each structure was placed on micrometric translation stages and subjected to controlled stretching. The total length of the sensing head was subjected to strain. To determine the wavelength variations, the wavelength change of the several labeled peaks in Figure 5 was monitored. Their mean value was used since no strain sensitivity dependence on wavelength was observed.

Figure 6 shows that the strain sensitivity increases when the taper diameter decreases. This behavior is expected [14]. The sensing head is composed by an SCF section combined with a fused taper. The strain gauge of the sensing head has a total length of $L_{\text {Total }}=L_{\text {taper }}+L_{\text {fiber }}$. If strain $\left(\varepsilon_{\text {taper }}\right)$ is applied to the taper structure, at constant temperature, the wavelength will be shifted by $\Delta \lambda_{\text {taper }}$ according to:

$$
\Delta \lambda_{\text {taper }}=\kappa_{\varepsilon(\text { taper })} \varepsilon_{\text {taper }}
$$

where $\kappa_{\varepsilon(\text { taper })}$ is a constant characteristic of the fiber material, which can be easily determined experimentally by analyzing the variation of the wavelength as a function of strain, at constant temperature.

However, if strain is applied to the entire sensor, then an unequal load of stress will appear along each section of the fiber depending on the mechanical resistance. In particular, strain loads applied to the SCF and the fused taper are related according to:

$$
\varepsilon_{\text {fiber }} E A_{\text {fiber }}=\varepsilon_{\text {Taper }} E A_{\text {Taper }}
$$

where $E$ is the Young modulus of the sensor material and $A_{i}$ ( $i=$ fiber or taper) is the cross-sectional area of the suspended core in the untapered and tapered regions respectively. It is assumed that the mechanical properties of the material in both suspended core and taper regions are the same. Consequently, the strain applied to the two sensor sections and the cladding diameters are related according to:

$$
\frac{\varepsilon_{\text {fiber }}}{\varepsilon_{\text {taper }}}=\frac{d_{\text {taper }}^{2}}{d_{\text {fiber }}^{2}}
$$

where $d_{f i b e r}$ and $d_{\text {taper }}$ are the cladding diameters of the SCF and taper, respectively. The longitudinal strain of the fused taper and piece of fiber are given by:

$$
\varepsilon_{\text {taper }}=\frac{\Delta L_{\text {taper }}}{L_{\text {taper }}} \quad \varepsilon_{\text {fiber }}=\frac{\Delta L_{\text {fiber }}}{L_{\text {fiber }}}
$$

where $L_{\text {taper }}$ and $L_{\text {fiber }}$ are the length of the sensor sections and $\Delta L_{\text {taper }}$ and $\Delta L_{\text {fiber }}$ are the extension of the taper and the fiber, respectively. By definition, the total longitudinal strain of the sensing head is given by:

$$
\varepsilon=\frac{\Delta L_{\text {taper }}+\Delta L_{\text {fiber }}}{L_{\text {taper }}+L_{\text {fiber }}}
$$

Combining equations (3)-(5), it is possible to derive the relationship between the strain applied to the whole sensor and the taper as:

$$
\varepsilon_{\text {taper }}=\frac{L_{\text {taper }}+L_{\text {fiber }}}{L_{\text {fiber }} \frac{d_{\text {taper }}^{2}}{d_{\text {fiber }}^{2}}+L_{\text {taper }}} \varepsilon
$$


Substituting equation (6) into equation (1), the wavelength shift can be rewritten as:

$$
\Delta \lambda_{\text {taper }}=\kappa_{\varepsilon(\text { taper })} \frac{L_{\text {taper }}+L_{\text {fiber }}}{L_{\text {fiber }} \frac{d_{\text {taper }}^{2}}{d_{\text {fiber }}^{2}}+L_{\text {taper }}} \varepsilon
$$

From equation (7) we can write the sensitivity as:

$$
\kappa=\kappa_{\varepsilon(\text { taper })} \frac{L_{\text {taper }}+L_{\text {fiber }}}{L_{\text {fiber }} \frac{d_{\text {taper }}^{2}}{d_{\text {fiber }}^{2}}+L_{\text {taper }}}
$$

From equation (7), since $d_{\text {taper }}<d_{f i b e r}$, as $d_{\text {taper }}$ decreases the strain sensitivity increases, which is confirmed by the results shown in Figure 6 (a). The obtained mean strain sensitivity to taper size was $(2.7 \pm 0.1) \times 10^{-2} \operatorname{strain}^{-1}$. From $120 \mu \mathrm{m}(-$ $1.73 \mathrm{pm} / \mu \varepsilon$ ) to $70 \mu \mathrm{m}(-3.12 \mathrm{pm} / \mu \varepsilon$ ), strain sensitivity increased approximately $80 \%$ (see Figure 6 ).

SCF tapers were also subjected to temperature variations but no sensitivity to such variations was observed. Figure 7 presents the spectral response of the $70 \mu \mathrm{m}$-SCF taper at $30^{\circ} \mathrm{C}$ and $70{ }^{\circ} \mathrm{C}$ where no discernable wavelength shift can be detected. This result is expected due to the properties of the SCF. The fiber is made of pure silica and its thermo-optic coefficient is very low.

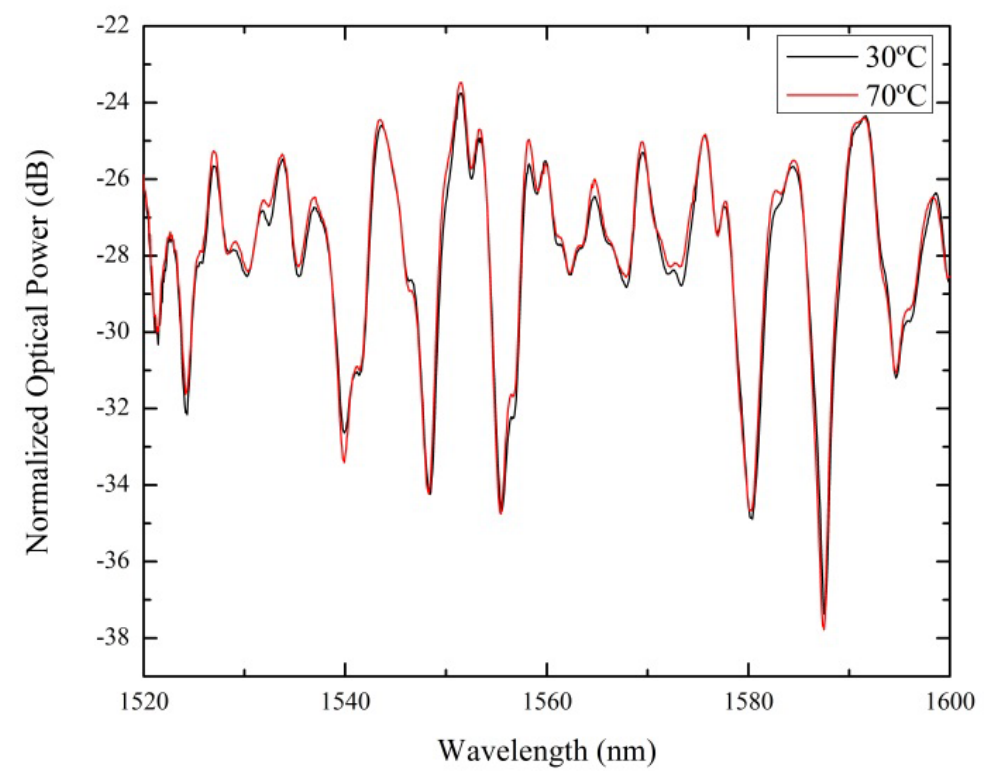

Figure 7. Temperature dependence of the $70 \mu \mathrm{m}$ SCF taper.

By changing the surrounding medium's refractive index, the tapers were characterized as possible optical fiber refractometric sensors. No spectral change was observed and they were deemed insensitive to external index of refraction variations. The sensing region is basically the core and it is protected from the surrounding medium by the air holes and cladding. 


\section{CONCLUSIONS}

Tapers from $70 \mu \mathrm{m}$ to $120 \mu \mathrm{m}$ were produced on an originally $125 \mu \mathrm{m}$-diameter SCF and characterized. Simulations showed a large decrease in the number of modes when tapering the SCF. This was confirmed through the analysis of the spectral responses. The tapers were deemed insensitive to temperature and external refractive index but extremely sensitive to strain. Sensitivities of $-1.73 \mathrm{pm} / \mu \varepsilon$ and $-3.12 \mathrm{pm} / \mu \varepsilon$ for $120 \mu \mathrm{m}$ and $70 \mu \mathrm{m}$ taper structures, respectively, were obtained. An increase of strain sensitivity with decreasing taper diameter was expected from simulations and experimentally observed. It was also verified that even when the SCF was tapered down to $40 \mu \mathrm{m}$ the holes did not collapse.

\section{ACKNOWLEDGEMENTS}

The authors would like to thank Fundação Calouste Gulbenkian for the grant provided under the award "Prémio de Estímulo à Investigação" attributed to Ricardo Melo André.

\section{REFERENCES}

[1] J. D. Love, W. M. Henry, W. J. Stewart, R. J. Black, S. Lacroix, and F. Gonthier, "Tapered single-mode fibers and devices .1. adiabaticity criteria," Ieee Proceedings-J Optoelectronics 138(5), pp. 343-354, 1991.

[2] T. A. Birks and Y. W. Li, “The shape of fiber tapers," Journal of Lightwave Technology 10(4), pp. 432-438, 1992.

[3] L. M. Tong, R. R. Gattass, J. B. Ashcom, S. L. He, J. Y. Lou, M. Y. Shen, I. Maxwell, and E. Mazur, "Subwavelength-diameter silica wires for low-loss optical wave guiding," Nature 426(6968), pp. 816-819, 2003.

[4] T. E. Dimmick, G. Kakarantzas, T. A. Birks, and P. S. Russell, "Carbon dioxide laser fabrication of fused-fiber couplers and tapers," Applied Optics 38(33), pp. 6845-6848, 1999.

[5] G. Brambilla, V. Finazzi, and D. J. Richardson, "Ultra-low-loss optical fiber nanotapers," Optics Express 12(10), pp. 2258-2263, 2004.

[6] S. G. Leon-Saval, T. A. Birks, W. J. Wadsworth, P. S. J. Russell, and M. W. Mason, "Supercontinuum generation in submicron fibre waveguides," Optics Express 12(13), pp. 2864-2869, 2004.

[7] F. Xu, P. Horak, and G. Brambilla, "Optical microfiber coil resonator refractometric sensor," Optics Express 15(12), pp. 7888-7893, 2007.

[8] J. K. Chandalia, B. J. Eggleton, R. S. Windeler, S. G. Kosinski, X. Liu, and C. Xu, "Adiabatic coupling in tapered air-silica microstructured optical fiber," Ieee Photonics Technology Letters 13(1), pp. 52-54, 2001.

[9] S. T. Huntington, J. Katsifolis, B. C. Gibson, J. Canning, K. Lyytikainen, J. Zagari, L. W. Cahill, and J. D. Love, "Retaining and characterising nano-structure within tapered air-silica structured optical fibers," Optics Express 11(2), pp. 98-104, 2003.

[10] E. C. Magi, P. Steinvurzel, and B. J. Eggleton, "Tapered photonic crystal fibers," Optics Express 12(5), pp. 776-784, 2004.

[11] J. Villatoro, V. P. Minkovich, and D. Monzón-Hernández, "Temperature-independent strain sensor made from tapered holey optical fiber," Opt. Lett. 31, pp. 305-307, Feb 2006.

[12] B. Y. Kim, J. N. Blake, S. Y. Huang, and H. J. Shaw, "Use of highly elliptical core fibers for two-mode fiber devices," Opt. Lett. 12, pp. 729-731, Sep 1987.

[13] K. Okamoto, Fundamentals of optical waveguides, Elsevier, 2006.

[14] O. Frazão, S. F. O. Silva, A. Guerreiro, J. L. Santos, L. A. Ferreira, and F. M. Araújo, "Strain sensitivity control of fiber bragg grating structures with fused tapers," Appl. Opt. 46(36), pp. 8578-8582, 2007. 